\title{
Indicadores para la evaluación de las instituciones universitarias: validación a través del método Delphi
}

\author{
Adela García-Aracil*, Davinia Palomares-Montero*
}

Resumen: Evaluar el desempeño de las universidades es complicado ya que se trata de una institución multidimensional que desarrolla numerosas actividades en base a sus tres principales misiones: docencia, investigación y transferencia de conocimiento. En este contexto, diversas metodologías de evaluación de las universidades han sido propuestas en base al uso de indicadores, existiendo intensos debates acerca de qué indicadores son los más apropiados. Esta situación hace que resulte indispensable sistematizar la diversidad de indicadores para obtener una evaluación más rigurosa de las instituciones universitarias. El presente trabajo profundiza en esta cuestión, identificando un listado de indicadores relacionados con la evaluación de las universidades. El sistema de indicadores propuesto ha sido validado a través del método Delphi que ha permitido alcanzar cierto consenso entre los expertos participantes. La identificación realizada sirve de ayuda para tomar decisiones en torno a qué indicadores deben ser incluidos en los modelos de evaluación universitaria. Sin embargo, los resultados del estudio ponen de manifiesto que entre los gestores universitarios continúa habiendo discrepancias en cuanto a la identificación de los indicadores tanto a qué misión representan como en su tipología y grado de importancia.

Palabras clave: instituciones de educación superior, evaluación, sistemas de indicadores, método Delphi.

\section{Indicators for evaluating higher education institutions: validation through the Delphi method}

Abstract: The evaluation of higher education activities is complex given their multiple objectives, which focus activities on the three principal academic missions of teaching, research and knowledge transfer. Various methodologies using indicators for the evaluation of universities have been proposed in the professional literature and there is intense debate over which are the most appropriate. Therefore, it is necessary to establish criteria against which to judge the existing indicators. This paper contributes directly to both theory and practice by providing a list of prioritized critical indicators for the evaluation of higher education institutions. For that purpose, we use the Delphi technique to try to achieve some sort of consensus among a group of experts with in-depth knowledge of higher education evaluation. Our results show that among the panel of experts there is no consensus and no clear cut division on the identification of which

* INGENIO (CSIC-UPV), Universitat Politècnica de València. Valencia. Correo-e: agarcia@ingenio. upv.es; dpalomares@ingenio.upv.es.

Recibido: 25-05-2011; 2. ${ }^{a}$ versión: 17-07-2011; aceptado: 26-7-2011. 
indicators are the most effective for the evaluation of university missions, the type of indicator and their degree of importance in the evaluation process.

Keywords: higher education institutions, evaluation, indicator systems, Delphi method.

\section{Introducción}

La evaluación de la educación superior es un proceso complejo que puede llevarse a cabo desde diferentes enfoques en función de su finalidad. El presente trabajo se centra en el uso de sistemas de indicadores, dado que se considera que esta herramienta ayuda a detectar con rapidez las tendencias de los cambios en las necesidades de la sociedad, en las preferencias por determinados tipos de estudios universitarios y, en consecuencia, en la reasignación de recursos entre las partes del sistema educativo más necesitadas en cada momento (Sizer, 1982).

Los sistemas de indicadores surgieron cuando la evaluación por pares cobraba mayor protagonismo, siendo cada vez más utilizados en la evaluación de las Instituciones de Educación Superior (IES). De hecho, desde la segunda mitad de los años setenta han surgido multitud de propuestas de indicadores de muy diversa índole para evaluar a las IES en Europa, dado que ofrecen a los gestores universitarios y a los legisladores información veraz de la actividad de la universidad (Kells, 1991; Blank, 1993; Mollas-Gallart, 2002; Palomares-Montero y otros, 2008; Buela Casal y otros, 2009).

Muchas de las propuestas de indicadores desarrolladas hacen alusión a los recursos que dispone la institución para desempeñar sus misiones. Estos indicadores se refieren principalmente a los recursos humanos y económicos, mientras que los recursos físicos son menos frecuentes (AQU, 2007; CIHE, 2007). Adicionalmente, dado que gran parte de los recursos que dispone la universidad son los mismos para realizar las diferentes actividades sin ningún tipo de diferenciación, algunas propuestas recomiendan desarrollar indicadores que tengan en cuenta el carácter multidimensional de la universidad, y no tanto indicadores sintéticos (Buela-Casal y otros, 2007; Fundación CyD, 2011). Por otra parte, otras propuestas informan de los resultados alcanzados por la universidad, observándose con mayor frecuencia resultados relacionados con la actividad de docencia y de investigación, si bien también han aparecido propuestas de indicadores orientadas a la medición de los resultados relacionados con la transferencia de conocimiento (Molas-Gallart, 2002; Hernández Armenteros, 2003; Buela Casal y otros, 2009).

Estas baterías de indicadores están siendo utilizadas por las agencias de evaluación y acreditación de la educación superior, por organismos interesados en el establecimiento de rankings universitarios y por las propias universidades preocupadas por mejorar su funcionamiento (Buela Casal y otros, 2009; ENQA, 2009). Sin embargo, el método de evaluación y la tipología de los indicadores aplicados en cada caso varía en función de qué es lo que se pretende medir (Buesa y otros, 2009). Por tanto, la confección y la elección del indicador no es trivial (Castro y 
Buela-Casal, 2008; Buesa y otros, 2009), por lo que debería establecerse un marco teórico común que aportara fundamentos y esclareciera la conceptualización de los indicadores.

La tarea que conlleva analizar y estructurar la diversidad de estos indicadores es realmente compleja, pues su uso no es únicamente una actividad técnica o neutra, sino que supone juicios de valor y responde a una serie de objetivos predeterminados (Molas-Gallart y Castro-Martínez, 2007; Lepori y otros, 2008; García-Aracil y Palomares-Montero, 2010). Esta es la razón de que, hasta la fecha, no se haya alcanzado un consenso entre los diferentes organismos involucrados en la definición de un sistema común aceptado por todos los implicados y en la forma de cuantificarlos. Sin embargo, a pesar de las dificultades y limitaciones encontradas, en los próximos años debería haber movimientos hacia la consolidación de los mismos, como aseguramiento en el alcance de la calidad (Bonaccorsi y otros, 2007), en base a principios de relevancia, viabilidad y fiabilidad (Oakes, 1989) para que permitan a su vez realizar comparaciones a lo largo del tiempo y entre instituciones (D'Este y otros, 2009). En este sentido, dada la proliferación de indicadores que se han propuesto para la evaluación conjunta de la institución universitaria y para cada una de sus tres principales misiones (docencia, investigación y transferencia de conocimiento), resulta pertinente reflexionar acerca del procedimiento seguido en el diseño, elaboración y selección de los mismos.

El presente trabajo pretende salvar el inconveniente derivado de la falta de acuerdo citado anteriormente. El interés del análisis se centra en alcanzar, entre un grupo de expertos, el consenso acerca de cuáles son los indicadores apropiados para evaluar la actividad y el rendimiento de las universidades. El estudio permitirá, asimismo, caracterizar un listado de indicadores en base a la misión universitaria a la que se refieren (docencia, investigación, transferencia de conocimiento, o la combinación de ellas ya que las universidades son instituciones multidimensionales), a su tipología (input, proceso, output, o la combinación de éstos, dado que en ocasiones existen sinergias entre los distintos tipos) y su importancia en el proceso de evaluación. Esta propuesta de caracterización de los indicadores permitirá un mejor análisis a la hora de realizar distintos ejercicios de evaluación de las IES (institucines de enseñanza superior), pues ofrece una visión global y flexible de las actividades de las IES.

Para obtener el resultado que pretende este estudio, utilizamos el método Delphi que ha sido aplicado con éxito en el ámbito de la educación superior para desarrollar y validar el uso de indicadores en la evaluación de los repositorios institucionales (Kim y Kim, 2008), en las políticas de evaluación institucional (Santana Armas, 2007), para explorar las opiniones sobre el rol de la educación superior en relación con el desarrollo humano y social (Lobera, 2008), en procesos de acreditación (GUNI, 2007) y en la financiación (Escrigas, 2006).

El método Delphi es un proceso repetitivo donde un grupo de expertos es consultado al menos dos veces sobre la misma cuestión, de manera que los participantes pueden reconsiderar su respuesta en base a la información que reciben 
del resto de expertos que forman el panel. Es un método que ofrece las ventajas propias de las respuestas grupales, eludiendo las limitaciones derivadas de la asistencia presencial (Geist, 2010). De esta manera, los participantes pueden ofrecer una opinión razonada e independiente, dado que no sienten la presión social o la influencia personal de sus pares (Dalkey y Helmer, 1963; Landeta, 2006). Además, permite mantener el anonimato entre los participantes. Otra ventaja del método Delphi es su eficiencia y flexibilidad ya que las nuevas tecnologías permiten obtener respuestas de individuos que se encuentran en zonas geográficas lejanas (Okoli y Pawlowski, 2004; Geist, 2010).

Sin embargo, el método Delphi presenta también limitaciones. Por un lado, el experto participante debe estar comprometido con el proceso de comunicación, debe tener habilidades en comunicación escrita y disponer de tiempo para responder (Gustafso y otros, 1973). Por tanto, se trata de un método que consume más tiempo que otros métodos de comunicación grupal, de ahí las elevadas tasas de abandono que se registran en su aplicación (Landeta, 2006). Otra limitación es que existe cierto grado de incertidumbre al no poder controlar directamente la forma en que finalmente se rellenaron los cuestionarios (Landeta, 2006). A pesar de estas limitaciones, el método Delphi ha sido ampliamente extendido y reconocido como un instrumento que facilita la toma de decisiones (Dalkey y Helmer, 1963; Landeta, 2006).

La puesta en marcha del método Delphi requiere del diseño y elaboración de un cuestionario. En nuestro estudio, con el objetivo de alcanzar el consenso acerca de cuáles son los indicadores apropiados para evaluar la actividad de las universidades, proponemos un listado comprensivo de indicadores cualitativos y cuantitativos, extraído de una revisión bibliográfica de indicadores previa (para más detalle véase Palomares-Montero y otros, 2008), para que un grupo de expertos exprese su opinión sobre ellos. Estos indicadores podrían ser aplicados en distintos contextos geográficos y ofrecer racionalidad en las evaluaciones de las IES.

\section{Metodología y diseño de la muestra}

Se han desarrollado diferentes variantes del método Delphi en función del tipo de problema a resolver y el propósito de la investigación (Okoli y Pawlowski, 2004; von der Gracht, 2008). Por un lado, el Delphi clásico se vincula a asuntos técnicos y busca el consenso entre un grupo homogéneo de expertos para conseguir la predicción más real posible. Por otro lado, el Delphi político está dirigido al análisis de situaciones sociales y sus objetivos principales son asegurar que todas las alternativas posibles se han puesto en el cuestionario para su consideración y estimar el impacto y las consecuencias de una opción particular. Por otra parte, el Delphi para la toma de decisión se caracteriza por la forma en que se constituye el panel de expertos, teniendo en cuenta su posición en la estructura jerárquica de la institución. En los tres casos, el método Delphi posibilita 
formar un grupo de expertos teniendo en cuenta principalmente su motivación por el proceso y su nivel de pericia, ya que no pretende trabajar con muestras representativas (Landeta, 2006).

En este estudio hemos aplicado el Delphi para la toma de decisiones, quedando el panel de expertos compuesto por altos cargos académicos y de gestión en la universidad española con experiencia en temas de evaluación universitaria tales como Rectores, Gerentes, Vicerrectores y Directores de las Oficinas de Transferencia de Resultados de Investigación (OTRI) (véase tabla I).

Para conocer la opinión del panel de expertos y tratar de alcanzar cierto grado de consenso entre ellos, se diseñó un cuestionario, previamente testado, que recogía una batería de indicadores para evaluar a las universidades (apéndice 1). El cuestionario estaba dividido en tres bloques. El primer bloque trataba de recoger información relacionada con las variables socio-demográficas para caracterizar el perfil del experto. El segundo bloque recogía seis ítems en los que se pedía al experto que manifestara su opinión respecto al uso de indicadores en la evaluación de universidades. En el tercer bloque se solicitaba a los expertos que identificaran del listado de indicadores presentados, cuáles eran más apropiados para: i) analizar las misiones de la universidad (docencia, investigación y transferencia de conocimiento); ii) señalar la tipología del indicador (input, proceso u output), e iii) indicar su grado de importancia en el proceso de evaluación.

Con el propósito de dar formalidad institucional al cuestionario y así lograr una mayor cuota de participación e implicación de los sujetos, se contactó con distintos organismos oficiales, como son la Conferencia de Rectores de las Universidades Españolas (CRUE) y la RedOTRI, para solicitar la participación expresa del experto en el estudio. Este apoyo institucional fue requerido para tratar de minimizar la incertidumbre derivada de la falta de control en la cumplimentación de los cuestionarios, ya que el contacto entre la CRUE, la RedOTRI y el panel de expertos fue directo.

El desarrollo del método de trabajo se llevó a cabo en dos rondas durante los meses de Mayo y Octubre de 2009 mediante el uso del correo electrónico. En la primera de ellas, fueron 45 los expertos que decidieron tomar parte del estudio para expresar sus opiniones y juicios con respecto a las preguntas planteadas en la encuesta. Posteriormente, las respuestas a esta primera ronda fueron analizadas, obteniendo datos estadísticos para cada ítem del cuestionario a partir de la distribución de las respuestas (mediana, cuartil inferior, cuartil superior, mínimo y máximo). Dicha información se utilizó como base para el cuestionario de la segunda ronda, con el objetivo de disminuir la dispersión de las opiniones y precisar la opinión media consensuada, lo cual permitió, por un lado, mejorar la formulación de varias preguntas con el fin de garantizar que todos los expertos respondían verdaderamente a la misma cuestión; y, por otro lado, hizo posible que los argumentos y reflexiones realizados fueran conocidos por los demás expertos y valorados en sus respectivas respuestas, de forma que el panelista pudiera decidir si mantenía su respuesta u optaba por modificarla en base a la respuesta del grupo. 
La segunda ronda del cuestionario Delphi contó con la colaboración de 37 de los expertos que participaron en la primera ronda. La Tabla I muestra la composición del panel de expertos en ambas rondas. Se observa que el grupo más representado es el correspondiente a los Vicerrectores de Investigación, seguido de los Directores de las OTRI, los Vicerrectores de Planificación Académica, los Gerentes, los Vicerrectores de Planificación Estratégica y Calidad, los Vicerrectores de Planificación Económica y el Rector. Por lo tanto, tras la segunda ronda, ocho panelistas quedaron fuera del proceso, produciéndose el abandono propio de estudios en los que se requiere consultar en más de una ocasión al mismo sujeto; asimismo, se puede asumir que el número de expertos es aceptable teniendo en cuenta que el margen sugerido para el buen uso del método es de 7 a 50 personas (Landeta, 1999).

\section{TABLA I}

Composición del panel de expertos acorde a su perfil (1. ${ }^{a}$ y $2{ }^{a}$ ronda)

\begin{tabular}{l|c|c|c|c}
\hline \multirow{2}{*}{\multicolumn{1}{c|}{ Perfil }} & \multicolumn{2}{c|}{$\mathbf{1 .}^{\mathbf{a}}$ Ronda } & \multicolumn{2}{c}{$\mathbf{2 .}^{\mathbf{a}}$ Ronda } \\
\cline { 2 - 5 } & $\mathbf{N}^{\mathbf{o}}$ & $\mathbf{\%}$ & $\mathbf{N}^{\mathbf{o}}$ & \multicolumn{1}{c}{$\%$} \\
\hline Vicerrector de Investigación & 12 & 26,7 & 12 & 32,4 \\
\hline Director OTRI & 11 & 24,4 & 9 & 24,3 \\
\hline Vicerrector de Planificación Académica & 8 & 17,8 & 7 & 18,9 \\
\hline Gerente & 5 & 11,1 & 4 & 10,8 \\
\hline Vicerrector de Planificación Estratégica y Calidad & 4 & 8,9 & 2 & 5,4 \\
\hline Vicerrector de Planificación Económica & 3 & 6,7 & 2 & 5,4 \\
\hline Rector & 2 & 4,4 & 1 & 2,7 \\
\hline Total & 45 & 100,0 & 37 & 100,0 \\
\hline
\end{tabular}

Fuente: elaboración propia.

En la siguiente sección mostramos los resultados obtenidos en la aplicación del método Delphi. Este análisis nos ofrecerá la oportunidad de identificar aquellos indicadores que mejor describan el ethos de la universidad.

\section{Resultados}

En este apartado, se presentan las respuestas de los expertos en relación a la identificación de los indicadores, acorde a la misión, la tipología y nivel de importancia de los mismos para ser utilizados en la evaluación de las universidades. 
Una de las formas de identificar el consenso entre las opiniones de los expertos es a través de la ponderación de las respuestas y la corrección de los sesgos. Así, tras la primera ronda se analizaron las respuestas y se reenviaron al grupo de panelistas para que los participantes pudieran repensar sus contestaciones en base a la opinión general del grupo. En nuestro caso, como la selección de los expertos no fue aleatoria, sino en base al elevado grado de especialización en temas de evaluación de las universidades, no se consideró necesario ofrecer al experto la posibilidad de autoevaluar su grado de conocimiento del tema a consultar, lo que habría permitido dar una ponderación diferente en los resultados grupales según el grado de conocimiento manifestado. En estas circunstancias, el consenso se definió siguiendo la regla aplicada por otros autores (Rowe y Wright, 1999; Landeta, 2006): cuando un ítem en una pregunta con dos alternativas acumula el $70 \%$ de las respuestas o cuando un ítem en una pregunta de respuesta múltiple acumula al menos el 50 \% de las respuestas. Por tanto, el consenso entre los expertos se analiza en base a la distribución de frecuencias donde la respuesta individual se expresa como porcentaje del total de frecuencias sobre el $100 \%$.

La tabla II recoge la distribución porcentual de las respuestas de los expertos con respecto a la identificación de los indicadores propuestos para la evaluación de las tres misiones principales de la universidad (docencia, investigación y transferencia de conocimiento). Dado que la universidad desempeña diferentes roles y misiones de forma conectada e inseparable (Gibbons, 1999; Martin y Etzkowitz, 2000; European Commission, 2005; Laredo, 2007), formulamos la pregunta del cuestionario de manera que permitiera posibles respuestas múltiples con el fin de capturar la multidimensionalidad de la IES; éstas vienen recogidas en cada una de las columnas de la tabla II, que son: (D) Docencia; (I) Investigación; (TC) Transferencia de Conocimiento; (D-I) Docencia e Investigación; (D-TC) Docencia y Transferencia de Conocimiento; (I-TC) Investigación y Transferencia de Conocimiento; (D-I-TC) Docencia, Investigación y Transferencia de Conocimiento. En general, se puede decir que el consenso alcanzado es considerable: en 28 de los 40 indicadores presentados, un ítem agrupa más del $50 \%$ de las respuestas.

Podemos observar que los indicadores asociados con la evaluación de la docencia son: los ítems que recogen información relacionada con el número de estudiantes en $1 .^{\text {er }}$ y $2 .{ }^{\circ}$ ciclo y el número de ellos que realizan prácticas en empresas (ítems 1 y 2, respectivamente), el número de graduados (ítem 3), los ingresos medios de los graduados en su primer trabajo (ítem 8), el cociente de PDI por el número de estudiantes (ítem 11) y los resultados de la encuesta de evaluación de la labor docente del PDI (ítem 12).

Los indicadores relacionados con la evaluación de la investigación, según la opinión de los expertos, son: los ítems que recaban información relacionada con las tesis doctorales defendidas (ítem 17), las publicaciones en revistas indexadas, las citas que ha recibido una publicación y el porcentaje de PDI que publica en revistas indexadas (ítems 18, 19 y 20), los sexenios concedidos (ítem 23), la participación en redes científicas (ítem 24), los ingresos por proyectos nacionales y 
europeos y los fondos asignados por la universidad a los centros de investigación (items 26, 27 y 30).

Por lo que respecta a los indicadores asociados con la evaluación de la transferencia de conocimiento, los expertos señalan los siguientes: los ingresos recibidos por trabajos realizados a entidades con alto valor añadido (ítem 29), las licencias concedidas (ítem 34), el número de spin-off originadas por la I+D de la universidad (ítem 35), la participación en empresas que desarrollan actividades productivas basadas en la I+D universitaria (ítem 36), la existencia de incubadora y el número de empresas ubicadas en la misma (ítems 37 y 38).

Si centramos nuestra atención en las cuatro últimas columnas de la tabla II, se observa que, en base a la opinión de los expertos encuestados, determinados indicadores no hacen referencia exclusivamente a una misión, sino que pueden ser utilizados para referirse a varias misiones, dependiendo del contexto y del uso que se haga de ellos. En este sentido, los indicadores ligados al número de PDI dedicado exclusivamente a la investigación, al número de PAS de apoyo a la investigación, al número de contratos de investigación y la existencia de un parque científico y/o tecnológico (ítems 14, 15, 32 y 39) fueron catalogados como indicadores asociados tanto a la misión investigación como a la transferencia de conocimiento.

Asimismo, también aparecen indicadores relacionados a la vez con las tres misiones básicas de la universidad; éstos son el número de PDI y PAS y las infraestructuras universitarias (items 9, 10 y 40). Si bien, a tenor de las respuestas de los expertos, de la lista de indicadores mostrados, ninguno hace referencia a docencia e investigación, ni a docencia y transferencia de conocimiento al mismo tiempo. De la consideración de estos resultados, podríamos inferir que, de forma general, los expertos consideran la investigación y la transferencia de conocimiento como misiones complementarias, mientras la relación entre la docencia y las otras dos misiones parece ser independiente. Aunque se trata de un resultado extrapolado de la opinión de los expertos, éste debería ser contrastado dado que ofrecería una idea de la forma en que se podrían relacionar las diferentes misiones.

Por otra parte, cabe reseñar que existen indicadores para los que el consenso no ha sido alcanzado entre los expertos, como son: el número de estudiantes en cursos de postgrado, el número de estudiantes postgraduados, así como aquéllos que realizan actividades de I+D o los que están subvencionados por la empresa (ítems 4, 5, 6 y 7); el cociente entre PAS y PDI (ítem 13); las apariciones del PDI en artículos de periódico (ítem 16); las publicaciones en revistas profesionales o sus trabajos presentados en congresos internacionales (ítems 21 y 22); los ingresos por proyectos de investigación local y regional y los ingresos por actividades de formación bajo demanda (ítems 25 y 28); el presupuesto de las OTRI (ítem 31); y el número de patentes solicitadas (ítem 33).

Por tanto, se puede afirmar que los indicadores anteriores que han sido identificados de forma consensuada con una de las misiones de la universidad deberían ser aplicados en la evaluación de la misión de la universidad a la que hacen 
referencia; si no fuera de esta manera, podríamos decir, siguiendo la opinión de los expertos participantes en esta investigación, que el indicador está siendo mal utilizado ya que estaría midiendo una vertiente de la universidad que realmente no se pretende medir y, por tanto, los resultados de la evaluación no serían los adecuados.

\section{TABLA II}

Opinión de los expertos respecto a la identificación del indicador para la evaluación de las misiones universitarias (fila =100\%)*

\begin{tabular}{|c|c|c|c|c|c|c|c|}
\hline Ítem & $\mathbf{D}$ & $\mathbf{I}$ & TC & D-I & D-TC & I-TC & D-I-TC \\
\hline 1. $\mathrm{N}^{\mathrm{o}}$ de estudiantes en $1 .^{\mathrm{er}}$ y $2 .^{\circ}$ ciclo & 94,6 & - & - & 5,4 & - & 一 & - \\
\hline 2. $\mathrm{N}^{\mathrm{o}}$ estudiantes $1{ }^{\mathrm{er}}$ y $2{ }^{\circ}$ ciclo con prácticas & 51,4 & - & 8,1 & - & 37,8 & 2,7 & - \\
\hline 3. $\mathrm{N}^{\mathrm{o}}$ de graduados & 83,8 & - & 2,7 & 5,4 & 5,4 & - & 2,7 \\
\hline 4. $\mathrm{N}^{\circ}$ de estudiantes en cursos de postgrado & 40,5 & 8,1 & 2,7 & 40,5 & 5,4 & - & 2,7 \\
\hline 5. $\mathrm{N}^{\mathrm{o}}$ de postgraduados & 32,4 & 16,2 & 2,7 & 35,1 & 13,5 & - & - \\
\hline 6. $\mathrm{N}^{\mathrm{o}}$ de postgraduados que participan en actividades de I+D & 2,7 & 43,2 & - & 5,4 & - & 40,5 & 8,1 \\
\hline 7. $\mathrm{N}^{\mathrm{o}}$ de postgraduados subvencionados por las empresas & 8,3 & 8,3 & 33,3 & - & 11,1 & 36,1 & 2,8 \\
\hline 8. Ingresos medios de los graduados en su 1 . $^{\text {er }}$ trabajo & 50,0 & - & 33,3 & - & 16,7 & - & - \\
\hline 9. $N^{\circ}$ de Personal Docer & - & 10,8 & - & 21,6 & - & 8,1 & 59,5 \\
\hline 10. $\mathrm{N}^{\mathrm{o}}$ de Personal de Administración y Servicios (PAS) & 8,6 & 2,9 & - & 34,3 & - & - & 54,3 \\
\hline 11. Cociente PDI por número de estudiantes & 75,0 & 2,8 & - & 11,1 & - & 一 & 11,1 \\
\hline 12. Resultados Encuesta de Evaluación de la labor docente & 100,0 & - & - & - & - & - & - \\
\hline 13. Cociente PAS por PDI & 6,1 & 12,1 & - & 33,3 & - & 3,0 & 45,5 \\
\hline 14. $N^{o}$ de PDI dedicado exclusivamente a la investigación & - & 37,8 & - & - & - & 59,5 & 2,7 \\
\hline 15. $N^{\circ}$ de PAS de apoyo a la investigación & - & 29,7 & 5,4 & - & - & 64,9 & - \\
\hline 16. $\mathrm{N}^{\circ}$ de apariciones del PDI en artículos de periódicos & 2,8 & 11,1 & 41,7 & 13,9 & - & 11,1 & 19,4 \\
\hline 17. $\mathrm{N}^{\mathrm{o}}$ de tesis doctorales defendidas & - & 54,1 & - & 21,6 & - & 18,9 & 5,4 \\
\hline 18. $\mathrm{N}^{\circ}$ de publicaciones en revistas indexadas & - & 72,2 & - & 一 & - & 22,2 & 5,6 \\
\hline 19. $\mathrm{N}^{\circ}$ de citas que ha recibido una publicación & 一 & 72,2 & - & - & - & 22,2 & 5,6 \\
\hline 20. \% PDI que produce publicaciones en revista indexada & - & 69,6 & - & - & - & 26,1 & 4,3 \\
\hline 21. $\mathrm{N}^{\circ}$ de publicaciones en revistas profesionales & - & 13,5 & 35,1 & 2,7 & - & 43,2 & 5,4 \\
\hline 22. $\mathrm{N}^{\circ}$ de trabajos presentados a congresos internacionales & - & 48,6 & - & - & - & 40,5 & 10,8 \\
\hline 23. $\mathrm{N}^{\circ}$ de sexenios concedidos & - & 81,1 & - & 5,4 & - & 13,5 & - \\
\hline 24. Participación en redes científicas & 一 & 67,6 & - & 5,4 & 一 & 18,9 & 8,1 \\
\hline 25. Ingresos por proyectos investigación local y regional & 一 & 48,6 & 5,4 & - & - & 45,9 & - \\
\hline
\end{tabular}


TABLA II (continuación)

\begin{tabular}{|c|c|c|c|c|c|c|c|}
\hline Ítem & $\mathbf{D}$ & $\mathbf{I}$ & TC & D-I & D-TC & I-TC & D-I-TC \\
\hline 26. Ingresos por proyectos investigación nacional & - & 59,5 & 2,7 & - & - & 37,8 & - \\
\hline 27. Ingresos por proyectos investigación europea & - & 54,1 & 2,7 & - & 2,7 & 40,5 & - \\
\hline 28. Ingresos por actividades de formación bajo demanda & 29,7 & - & 21,6 & - & 45,9 & 2,7 & - \\
\hline 29. Ingresos por trabajos a entidades con alto valor añadido & - & 2,7 & 73,0 & - & 2,7 & 21,6 & - \\
\hline 30. Fondos de la universidad a centros de investigación & - & 68,6 & 5,7 & - & - & 22,9 & 2,9 \\
\hline 31. Presupuesto de las OTRI & - & 11,4 & 42,9 & - & - & 45,7 & - \\
\hline 32. $\mathrm{N}^{\circ}$ contratos de investigación (universidades/entidades) & - & 14,3 & 14,3 & - & - & 71,4 & - \\
\hline 33. $\mathrm{N}^{\circ}$ de patentes solicitadas & - & 5,4 & 48,6 & - & - & 45,9 & - \\
\hline 34. $\mathrm{N}^{\circ}$ de licencias concedidas & - & 2,7 & 62,2 & - & - & 35,1 & - \\
\hline $\begin{array}{l}\text { 35. No de Spin-off originadas por la I+D de la Universi- } \\
\text { dad }\end{array}$ & - & - & 75,7 & - & - & 24,3 & - \\
\hline 36. Actividades empresariales basadas en I+D universitaria & - & 2,7 & 78,4 & - & - & 18,9 & - \\
\hline 37. Existencia de Incubadora & - & 2,8 & 83,3 & - & - & 13,9 & - \\
\hline 38. $\mathrm{N}^{\circ}$ de empresas en la Incubadora & - & 2,8 & 86,1 & - & - & 11,1 & - \\
\hline 39. Existencia de Parque Científico y/o Tecnológico & - & - & 45,9 & - & - & 51,4 & 2,7 \\
\hline 40. $\mathrm{M}^{2}$ de infraestructuras universitarias & 2,7 & - & - & 37,8 & 2,7 & 5,4 & 51,4 \\
\hline
\end{tabular}

Fuente: elaboración propia.

* (D) Docencia; (I) Investigación; (TC) Transferencia de Conocimiento; (D-I) Docencia e Investigación; (DTC) Docencia y Transferencia de Conocimiento; (I-TC) Investigación y Transferencia de Conocimiento; (D-I-TC) Docencia, Investigación y Transferencia de Conocimiento.

En la tabla III se recoge la opinión de los expertos respecto a la identificación de la tipología del indicador en la evaluación de las actividades de las IES, es decir, si se trata de un input (recurso), un proceso (actividad) o un output (resultado). Dada la falta de acuerdo en torno a la caracterización de algunos indicadores como input, proceso u output (Hanke y Leopoldseder, 1998; Bonaccorsi y Daraio, 2007), formulamos la pregunta del cuestionario de manera que permitiera posibles respuestas múltiples para capturar dicha diversidad en la tipología del indicador. Las posibles combinaciones vienen recogidas en cada una de las columnas de la tabla III, que son: (I) Input; (P) Proceso; (O) Output; (I-P) Input y Proceso; (I-O) Input y Output; (P-O) Proceso y Output; (I-P-O) Input, Proceso y Output. En esta ocasión, solo 17 de los 40 ítems recogen en alguna de sus categorías más del 50 \% de las observaciones.

Como se puede observar, 15 de los 17 indicadores en los que ha habido acuerdo entre los expertos han sido considerados como resultados de la actividad universitaria; éstos son: el número de graduados y postgraduados (ítems 3 y 5), los ingresos medios de los graduados en su primer trabajo (ítem 8), el número 


\section{TABLA III}

Opinión de los expertos respecto a la identificación de la tipología del indicador para la evaluación de las actividades universitarias (fila $=100 \%$ )*

\begin{tabular}{|c|c|c|c|c|c|c|c|}
\hline Ítem & $\mathbf{I}$ & $\mathbf{P}$ & $\mathbf{O}$ & I-P & I-O & P-O & I-P-O \\
\hline 1. N. ${ }^{\circ}$ de estudiantes en $1 .^{\text {er }}$ y $2 .^{\circ}$ ciclo & 35,1 & 27,0 & - & 24,3 & 2,7 & - & 10,8 \\
\hline 2. N. ${ }^{\circ}$ estudiantes $1 .^{\text {er }}$ y $2 .^{\circ}$ ciclo con prácticas & 3,0 & 36,4 & 9,1 & 9,1 & 3,0 & 27,3 & 12,1 \\
\hline 3. N. ${ }^{\circ}$ de graduados & - & 8,3 & 77,8 & - & - & 11,1 & 2,8 \\
\hline 4. N. ${ }^{\circ}$ de estudiantes en cursos de postgrado & 21,6 & 43,2 & - & 21,6 & 2,7 & - & 10,8 \\
\hline 5. N. ${ }^{\circ}$ de postgraduados & - & 8,1 & 78,4 & - & - & 8,1 & 5,4 \\
\hline 6. N. ${ }^{\circ}$ de postgraduados que participan en actividades de I+D & 10,8 & 29,7 & 13,5 & 2,7 & 2,7 & 35,1 & 5,4 \\
\hline 7. N. ${ }^{\circ}$ de postgraduados subvencionados por las empresas & 13,9 & 36,1 & 19,4 & - & 2,8 & 25,0 & 2,8 \\
\hline 8. Ingresos medios de los graduados en su $1 .^{\text {er }}$ trabajo & - & 2,9 & 97,1 & - & - & - & - \\
\hline 9. N. ${ }^{\circ}$ de Personal Docente Investigador (PDI) & 41,7 & 22,2 & - & 25,0 & - & 2,8 & 8,3 \\
\hline 10. N. ${ }^{\circ}$ de Personal de Administración y Servicios (PAS) & 47,2 & 27,8 & - & 13,9 & - & 5,6 & 5,6 \\
\hline 11. Cociente PDI por número de estudiantes & 33,3 & 44,4 & - & 16,7 & - & - & 5,6 \\
\hline 12. Resultados Encuesta de Evaluación de la lak & - & 56,5 & 21,7 & - & 4,3 & 17,4 & - \\
\hline 13. Cociente PAS por PDI & 27,0 & 40,5 & - & 24,3 & - & 5,4 & 2,7 \\
\hline 14. N. ${ }^{\circ}$ de PDI dedicado exclusivamente a la investigación & 35,1 & 18,9 & 2,7 & 13,5 & - & 10,8 & 18,9 \\
\hline 15. N. ${ }^{\circ}$ de PAS de apoyo a la investigación & 38,9 & 27,8 & - & 16,7 & 2,8 & 2,8 & 11,1 \\
\hline 16. N. ${ }^{\circ}$ de apariciones del PDI en artículos de periódicos & - & 8,6 & 71,4 & - & - & 17,1 & 2,9 \\
\hline 17. N. ${ }^{\circ}$ de tesis doctorales defendidas & - & 5,4 & 64,9 & - & - & 27,0 & 2,7 \\
\hline 18. N. ${ }^{\circ}$ de publicaciones en revistas indexadas & 一 & 3,0 & 66,7 & 一 & 一 & 30,3 & 一 \\
\hline 19. N. ${ }^{\circ}$ de citas que ha recibido una publicación & - & 6,1 & 75,8 & - & - & 18,2 & - \\
\hline 20. \% PDI que produce publicaciones en revista indexada & - & 17,4 & 52,2 & - & - & 30,4 & - \\
\hline 21. N. ${ }^{\circ}$ de publicaciones en revistas profesionales & - & 8,1 & 67,6 & - & - & 24,3 & - \\
\hline 22. N. ${ }^{\circ}$ de trabajos presentados a congresos internacionales & - & 5,4 & 59,5 & - & - & 35,1 & - \\
\hline 23. N. ${ }^{\circ}$ de sexenios concedidos & 5,6 & 16,7 & 58,3 & 2,8 & - & 13,9 & 2,8 \\
\hline 24. Participación en redes científicas & - & 48,6 & 21,6 & 5,4 & - & 21,6 & 2,7 \\
\hline 25. Ingresos por proyectos investigación local y regional & 18,9 & 10,8 & 16,2 & 10,8 & 18,9 & 16,2 & 8,1 \\
\hline 26. Ingresos por proyectos investigación nacional & 19,4 & 11,1 & 13,9 & 11,1 & 13,9 & 22,2 & 8,3 \\
\hline 27. Ingresos por proyectos investigación europea & 19,4 & 8,3 & 13,9 & 8,3 & 13,9 & 27,8 & 8,3 \\
\hline 28. Ingresos por actividades de formación bajo demanda & 10,8 & 8,1 & 37,8 & 10,8 & 10,8 & 13,5 & 8,1 \\
\hline 29. Ingresos por trabajos a entidades con alto valor añadido & 10,8 & - & 43,2 & 5,4 & 21,6 & 16,2 & 2,7 \\
\hline 30. Fondos de la universidad a centros de investigación & 34,3 & 31,4 & 5,7 & 17,1 & - & 5,7 & 5,7 \\
\hline 31. Presupuesto de las OTRI & 25,7 & 28,6 & 5,7 & 17,1 & 11,4 & 11,4 & - \\
\hline
\end{tabular}


TABLA III (continuación)

\begin{tabular}{l|c|c|c|c|c|c|c}
\hline \multicolumn{1}{c|}{ Ítem } & I & P & O & I-P & I-O & P-o & I-P-o \\
\hline 32. N. ${ }^{\circ}$ contratos de investigación (universidades/entidades) & 5,6 & 8,3 & 41,7 & 2,8 & 13,9 & 16,7 & 11,1 \\
\hline 33. N. ${ }^{\circ}$ de patentes solicitadas & - & 10,8 & 64,9 & - & - & 24,3 & - \\
\hline 34. N. ${ }^{\circ}$ de licencias concedidas & - & 83,8 & - & - & 2,7 & 13,5 & - \\
\hline 35. N. ${ }^{\circ}$ de Spin-off originadas por la I+D de la Universidad & - & 5,4 & 70,3 & - & 8,1 & 16,2 & - \\
\hline 36. Actividades empresariales basadas en I+D universitaria & - & 8,3 & 69,4 & - & 2,8 & 19,4 & - \\
\hline 37. Existencia de Incubadora & 11,8 & 20,6 & 41,2 & 8,8 & - & 14,7 & 2,9 \\
\hline 38. N. ${ }^{\circ}$ de empresas en la Incubadora & - & 17,6 & 61,8 & - & - & 17,6 & 2,9 \\
\hline 39. Existencia de Parque Científico y/o Tecnológico & 14,3 & 22,9 & 14,3 & 5,7 & - & 25,7 & 17,1 \\
\hline 40. M2 de infraestructuras universitarias & 42,9 & 28,6 & 2,9 & 17,1 & - & - & 8,6 \\
\hline
\end{tabular}

Fuente: elaboración propia.

* (I) Input; (P) Proceso; (O) Output; (I-P) Input y Proceso; (I-O) Input y Output; (P-O) Proceso y Output; (I-P-O) Input, Proceso y Output.

de apariciones del PDI en artículos de periódicos, las publicaciones en revistas indexadas, las citas que estas publicaciones reciben, el porcentaje de PDI que publica en estas revistas, el número de publicaciones en revistas profesionales y los trabajos presentados a congresos internacionales (ítems 16, 18, 19, 20, 21 y 22), las tesis doctorales defendidas (ítem 17), los sexenios concedidos (ítem 23), las patentes solicitadas, las spin-off originadas por la I+D de la universidad, la participación en empresas desarrollando actividades productivas basadas en la I+D universitaria y el número de empresas en la incubadora (ítems 33, 35, 36 y 38). Los dos indicadores restantes fueron considerados como procesos de la actividad universitaria y son: los resultados de la encuesta de evaluación de la actividad docente del PDI y las licencias concedidas (ítems 12 y 34).

Por otra parte, destacamos la variabilidad de opiniones con respecto a la consideración de la tipología en los indicadores referidos a los ingresos obtenidos por la universidad, bien a través de proyectos de investigación o bien por trabajos a entidades con alto valor añadido (ítem 25, 26, 27, 28 y 29). Se trata de un resultado que refleja el debate existente por considerar a los ingresos recursos $\mathrm{O}$ resultados de la investigación universitaria (Ahn y otros 1988; Cohn y Santos, 1989; Ahn y Seiford 1993); debate que queda sin respuesta en esta investigación.

Además, hemos podido observar que no ha habido consenso con respecto a la consideración de alguno de los indicadores mostrados, por ejemplo: el número de estudiantes en $1 .^{\text {er }}$ y $2 .^{\circ}$ ciclo y el número de estudiantes en $1 .^{\text {er }}$ y $2 .^{\circ}$ ciclo en cursos de postgrado (ítems 1 y 4). Algunos expertos los consideran solo indicadores de input, otros piensan que hacen referencia a procesos, mientras algunos creen que hacen referencia a inputs y procesos al mismo tiempo. Este resultado se encuentra acorde a otros trabajos que tratan de modelar la función 
de producción de la universidad, trabajos que no han alcanzado una propuesta definitiva para la selección de inputs u outputs (Beasley, 1995; Johnes y Johnes 1995; Worthington 2001; Bonaccorsi y otros, 2007). Asimismo, otros estudios consideran al número de estudiantes como un proceso (Sinuany-Stern y otros, 1994; Hanke y Leopoldseder, 1998), mientras otros como un indicador de input (Bonaccorsi y Daraio, 2007; García-Aracil, 2007).

En base a los resultados obtenidos en este apartado, se puede decir que la identificación de los resultados de las actividades universitarias genera un mayor acuerdo entre los expertos que, por el contrario, la identificación de recursos y procesos.

Finalmente, en la tabla IV se recoge la opinión de los expertos con respecto a la importancia que otorgan a cada uno de los indicadores en el proceso de evaluación de la institución universitaria, en una escala de 1 a 5, siendo (1) «nada importante» (no debe ser considerado en el proceso de evaluación) y (5) «muy importante» (esencial en el proceso de evaluación). Solo 12 de los 40 ítems propuestos recogen más del $50 \%$ de las respuestas en una de sus categorías.

\section{TABLA IV}

Importancia del indicador en la evaluación de las universidades (fila $=100 \%)$

\begin{tabular}{|c|c|c|c|c|c|}
\hline Ítem & $\begin{array}{l}\text { Nada } \\
\text { impor- } \\
\text { tante } 1\end{array}$ & 2 & $\begin{array}{c}\text { Neutro } \\
3\end{array}$ & 4 & $\begin{array}{c}\text { Muy } \\
\text { impor- } \\
\text { tante } 5\end{array}$ \\
\hline 1. $\mathrm{N}^{\mathrm{o}}$ de estudiantes en 1 er y $2 .^{\circ}$ ciclo & - & 2,7 & 21,6 & 32,4 & 43,2 \\
\hline 2. $\mathrm{N}^{\mathrm{o}}$ estudiantes 1 er y $2 .^{\circ}$ ciclo con prácticas en empresas & - & 8,3 & 22,2 & 36,1 & 33,3 \\
\hline 3. $\mathrm{N}^{\mathrm{o}}$ de graduados & - & - & 13,5 & 51,4 & 35,1 \\
\hline 4. $\mathrm{N}^{\circ}$ de estudiantes en cursos de postgrado & - & 5,4 & 16,2 & 37,8 & 40,5 \\
\hline 5. $\mathrm{N}^{\mathrm{o}}$ de postgraduados & - & 8,1 & 16,2 & 37,8 & 37,8 \\
\hline 6. $\mathrm{N}^{\mathrm{o}}$ de postgraduados que participan en actividades de $\mathrm{I}+\mathrm{D}$ & - & 2,7 & 18,9 & 51,4 & 27,0 \\
\hline 7. $\mathrm{N}^{\mathrm{o}}$ de postgraduados subvencionados por las empresas & - & 11,1 & 27,8 & 44,4 & 16,7 \\
\hline 8. Ingresos medios de los graduados en su 1er trabajo & 20,0 & 25,7 & 37,1 & 11,4 & 5,7 \\
\hline 9. $\mathrm{N}^{\mathrm{o}}$ de Personal Docente Investigador (PDI) & - & 2,7 & 27,0 & 35,1 & 35,1 \\
\hline 10. $\mathrm{N}^{\mathrm{o}}$ de Personal de Administración y Servicios (PAS) & - & 16,7 & 50,0 & 25,0 & 8,3 \\
\hline 11. Cociente PDI por número de estudiantes & 2,8 & 2,8 & 16,7 & 58,3 & 19,4 \\
\hline 12. Resultados Encuesta de Evaluación de la labor docente & - & 13,0 & 8,7 & 43,5 & 34,8 \\
\hline 13. Cociente PAS por PDI & - & 5,4 & 45,9 & 40,5 & 8,1 \\
\hline 14. $\mathrm{N}^{\mathrm{o}}$ de PDI dedicado exclusivamente a la investigación & - & 2,7 & 21,6 & 48,6 & 27,0 \\
\hline 15. $N^{\circ}$ de PAS de apoyo a la investigación & - & 8,1 & 21,6 & 45,9 & 24,3 \\
\hline 16. $\mathrm{N}^{\mathrm{o}}$ de apariciones del PDI en artículos de periódicos & 11,1 & 44,4 & 27,8 & 8,3 & 8,3 \\
\hline 17. $\mathrm{N}^{\circ}$ de tesis doctorales defendidas & - & - & 2,7 & 48,6 & 48,6 \\
\hline
\end{tabular}


TABLA IV (continuación)

\begin{tabular}{l|c|c|c|c|c}
\hline \multicolumn{1}{c|}{ Ítem } & $\begin{array}{c}\text { Nada } \\
\text { impor- } \\
\text { tante } \mathbf{1}\end{array}$ & $\mathbf{2}$ & $\begin{array}{c}\text { Neutro } \\
\mathbf{3}\end{array}$ & $\mathbf{4}$ & $\begin{array}{c}\text { Muy } \\
\text { impor- } \\
\text { tante 5 }\end{array}$ \\
\hline 18. No de publicaciones en revistas indexadas & - & - & 5,6 & 25,0 & 69,4 \\
\hline 19. No de citas que ha recibido una publicación & - & - & 8,3 & 38,9 & 52,8 \\
\hline 20. \% PDI que produce publicaciones en revista indexada & - & - & 4,3 & 47,8 & 47,8 \\
\hline 21. No de publicaciones en revistas profesionales & 2,7 & 8,1 & 45,9 & 37,8 & 5,4 \\
\hline 22. No de trabajos presentados a congresos internacionales & - & 21,6 & 35,1 & 32,4 & 10,8 \\
\hline 23. No de sexenios concedidos & 2,7 & 5,4 & 16,2 & 35,1 & 40,5 \\
\hline 24. Participación en redes científicas & - & 8,1 & 29,7 & 45,9 & 16,2 \\
\hline 25. Ingresos por proyectos investigación local y regional & - & - & 28,6 & 51,4 & 20,0 \\
\hline 26. Ingresos por proyectos investigación nacional & - & - & 8,1 & 51,4 & 40,5 \\
\hline 27. Ingresos por proyectos investigación europea & - & - & 5,4 & 29,7 & 64,9 \\
\hline 28. Ingresos por actividades de formación bajo demanda & - & 16,2 & 32,4 & 43,2 & 8,1 \\
\hline 29. Ingresos por trabajos a entidades con alto valor añadido & - & 8,1 & 21,6 & 43,2 & 27,0 \\
\hline 30. Fondos de la universidad a centros de investigación & 10,8 & 13,5 & 35,1 & 21,6 & 18,9 \\
\hline 31. Presupuesto de las OTRI & 5,4 & 5,4 & 32,4 & 35,1 & 21,6 \\
\hline 32. No contratos de investigación (universidades/entidades) & - & 2,8 & 11,1 & 58,3 & 27,8 \\
\hline 33. No de patentes solicitadas & 2,7 & - & 18,9 & 56,8 & 21,6 \\
\hline 34. No de licencias concedidas & - & - & 18,9 & 24,3 & 56,8 \\
\hline 35. No de Spin-off originadas por la I+D de la Universidad & - & 2,7 & 18,9 & 32,4 & 45,9 \\
\hline 36. Actividades empresariales basadas en I+D universitaria & - & 2,7 & 27,0 & 48,6 & 21,6 \\
\hline 37. Existencia de Incubadora & - & 5,6 & 33,3 & 36,1 & 25,0 \\
\hline 38. No de empresas en la Incubadora & - & 2,9 & 20,0 & 45,7 & 31,4 \\
\hline 39. Existencia de Parque Científico y/o Tecnológico & - & 5,4 & 18,9 & 35,1 & 40,5 \\
\hline 40. M2 de infraestructuras universitarias & 2,7 & 10,8 & 21,6 & 40,5 & 24,3 \\
\hline
\end{tabular}

Fuente: elaboración propia.

A tenor de las opiniones de los expertos, los indicadores que deben ser tenidos en cuenta en los procesos de evaluación de las universidades por ser considerados importantes o muy importantes son: el número de graduados y el número de postgraduados participando en actividades de I+D (ítems 3 y 6), el cociente de PDI por número de estudiantes (ítem 11), el número de publicaciones en revistas indexadas y las citas que reciben dichas publicaciones (ítems 18 y 19), los ingresos recibidos por proyectos tanto locales y regionales, como nacionales y europeos y el número de contratos de investigación (ítems 25, 26, 27 y 32), así como las patentes solicitadas y las licencias concedidas (ítems 33 y 34). 
Asimismo, resulta conveniente analizar las opiniones del panel de expertos también en aquellos casos en los que el consenso no ha sido alcanzado. Y es que, la suma agregada de las puntuaciones obtenidas en las categorías «importante» y "muy importante" revela valores que así considerados superan en trascendencia a otros que sí alcanzaron el consenso. En este sentido, destacan el número de tesis doctorales defendidas y el porcentaje de PDI que produce publicaciones en revistas indexadas (ítems 17 y 20) con valores superiores al $95 \%$ en dichas categorías. En general, si consideramos ambas categorías de forma conjunta (importante y muy importante), podríamos decir que también estos indicadores deberían ser utilizados en la evaluación de las IES dado que son considerados de forma generalizada como importantes.

A continuación, tomando como criterio de selección aquellos indicadores en los que se ha alcanzado el consenso en cuanto a su consideración como importantes en el proceso de evaluación de las universidades, observamos la opinión de los expertos respecto a su tipología y su misión, es decir, analizamos de forma cruzada ambas informaciones para dichos indicadores. En este caso, se observa que solo en tres de los 12 indicadores en los que se alcanzó el consenso en su consideración como importantes, se ha alcanzado el consenso respecto a la caracterización de su tipología y misión (véase tabla V). Los tres son considerados como indicadores de resultado; si bien el número de graduados (ítem 3) está relacionado con la docencia; las citas que una publicación recibe (ítem 19) es considerado de investigación y el número de licencias concedidas (ítem 34) se refiere a la transferencia de conocimiento. En el resto de estos 12 indicadores, a pesar de que los expertos han considerado que deben ser tenidos en cuenta en los procesos de evaluación, no se ha alcanzado el consenso suficiente con respecto a la catalogación de su tipología y su misión, por lo que se observa una diversidad importante en las respuestas.

\section{TABLA V}

Indicadores considerados como importantes en la evaluación de las universidades públicas acorde a su tipología y misión (fila = 100\%)

\begin{tabular}{l|c|c|c|c|c|c|c|c}
\hline \multirow{3}{*}{ Indicador } & \multirow{2}{*}{ Tipo+ } & \multicolumn{7}{c}{ Misión* } \\
\cline { 3 - 10 } & & D & I & TC & D-I & D-TC & I-TC & D-I-TC \\
\hline \multirow{3}{*}{$3 . N^{\circ}$ de graduados } & $\mathrm{I}$ & - & - & - & - & - & - & - \\
\cline { 2 - 10 } & $\mathrm{P}$ & 5,6 & - & - & 2,8 & - & - & - \\
\cline { 2 - 10 } & $\mathrm{O}$ & 69,4 & - & 2,8 & 2,8 & 2,8 & - & - \\
\cline { 2 - 9 } & $\mathrm{I}-\mathrm{P}$ & - & - & - & - & - & - & - \\
\cline { 2 - 9 } & I-O & - & - & - & - & - & - & - \\
\cline { 2 - 9 } & P-O & 5,6 & - & - & - & 2,8 & - & 2,8 \\
\hline & I-P-O & 2,8 & - & - & - & - & - & - \\
\hline
\end{tabular}


TABLA V (continuación)

\begin{tabular}{|c|c|c|c|c|c|c|c|c|}
\hline \multirow{2}{*}{ Indicador } & \multirow{2}{*}{ Tipo+ } & \multicolumn{7}{|c|}{ Misión* } \\
\hline & & D & $\mathbf{I}$ & TC & D-I & D-TC & I-TC & D-I-TC \\
\hline \multirow{7}{*}{$\begin{array}{l}\text { 6. } \mathrm{N}^{0} \text { de postgraduados que partici- } \\
\text { pan en actividades de } \mathrm{I}+\mathrm{D}\end{array}$} & I & - & 10,8 & - & - & - & - & - \\
\hline & $\mathrm{P}$ & 2,7 & 18,9 & - & 2,7 & - & 5,4 & - \\
\hline & $\mathrm{O}$ & - & - & - & - & - & 10,8 & 2,7 \\
\hline & I-P & - & 2,7 & - & - & - & - & - \\
\hline & I-O & - & - & - & - & - & - & 2,7 \\
\hline & P-O & - & 10,8 & - & 2,7 & - & 21,6 & - \\
\hline & I-P-O & - & - & - & - & - & 2,7 & 2,7 \\
\hline \multirow{7}{*}{$\begin{array}{l}\text { 10. } N^{o} \text { de Personal de Administración } \\
\text { y Servicios (PAS) }\end{array}$} & I & 5,7 & - & - & 8,6 & - & - & 31,4 \\
\hline & $\mathrm{P}$ & 2,9 & 2,9 & - & 17,1 & - & - & 5,7 \\
\hline & $\mathrm{O}$ & - & - & - & - & - & - & - \\
\hline & I-P & - & - & - & 5,7 & - & - & 8,6 \\
\hline & I-O & - & - & - & - & - & - & - \\
\hline & $\mathrm{P}-\mathrm{O}$ & - & - & - & - & - & - & 5,7 \\
\hline & I-P-O & - & - & - & 2,9 & - & - & 2,9 \\
\hline \multirow{7}{*}{$\begin{array}{l}\text { 11. Cociente PDI por número de es- } \\
\text { tudiantes }\end{array}$} & I & 27,8 & - & - & 5,6 & - & - & - \\
\hline & P & 33,3 & 2,8 & - & 5,6 & - & - & 2,8 \\
\hline & $\mathrm{O}$ & - & - & - & - & - & - & - \\
\hline & I-P & 13,9 & - & - & - & - & - & 2,8 \\
\hline & I-O & - & - & - & - & - & - & - \\
\hline & $\mathrm{P}-\mathrm{O}$ & - & - & - & - & - & - & - \\
\hline & I-P-O & - & - & - & - & - & - & 5,6 \\
\hline \multirow{7}{*}{$\begin{array}{l}\text { 18. } \mathrm{N}^{\mathrm{o}} \text { de publicaciones en revistas } \\
\text { indexadas }\end{array}$} & I & - & - & - & - & - & - & - \\
\hline & $\mathrm{P}$ & - & 3,0 & - & - & - & - & - \\
\hline & $\mathrm{O}$ & - & 45,4 & - & - & - & 15,2 & 6,1 \\
\hline & I-P & - & - & - & - & - & - & - \\
\hline & $\mathrm{I}-\mathrm{O}$ & - & - & - & - & - & - & - \\
\hline & $\mathrm{P}-\mathrm{O}$ & - & 21,2 & - & - & - & 9,1 & - \\
\hline & I-P-O & - & - & - & - & - & - & - \\
\hline \multirow{7}{*}{$\begin{array}{l}\text { 19. } \mathrm{N}^{0} \text { de citas que ha recibido una } \\
\text { publicación }\end{array}$} & I & - & - & - & - & - & - & - \\
\hline & P & - & 3,0 & - & - & - & 3,0 & - \\
\hline & $\mathrm{O}$ & - & 54,5 & - & - & - & 15,2 & 6,1 \\
\hline & I-P & - & - & - & - & - & - & - \\
\hline & I-O & - & - & - & - & - & - & - \\
\hline & $\mathrm{P}-\mathrm{O}$ & - & 12,1 & - & - & - & 6,1 & - \\
\hline & I-P-O & - & - & - & - & - & - & - \\
\hline
\end{tabular}


TABLA V (continuación)

\begin{tabular}{|c|c|c|c|c|c|c|c|c|}
\hline \multirow{2}{*}{ Indicador } & \multirow{2}{*}{ Tipo+ } & \multicolumn{7}{|c|}{ Misión* } \\
\hline & & D & I & TC & D-I & D-TC & I-TC & D-I-TC \\
\hline \multirow{7}{*}{$\begin{array}{l}\text { 25. Ingresos por proyectos investiga- } \\
\text { ción local y regional }\end{array}$} & I & - & 16,2 & 2,7 & - & - & - & - \\
\hline & $\mathrm{P}$ & - & 8,1 & - & - & - & 2,7 & - \\
\hline & $\mathrm{O}$ & - & - & 2,7 & - & - & 13,5 & - \\
\hline & $\mathrm{I}-\mathrm{P}$ & - & 10,8 & - & - & - & - & - \\
\hline & $\mathrm{I}-\mathrm{O}$ & - & 8,1 & - & - & - & 10,8 & - \\
\hline & $\mathrm{P}-\mathrm{O}$ & - & 2,7 & - & - & - & 13,5 & - \\
\hline & $\mathrm{I}-\mathrm{P}-\mathrm{O}$ & - & 2,7 & - & - & - & 5,4 & - \\
\hline \multirow{7}{*}{$\begin{array}{l}\text { 26. Ingresos por proyectos investiga- } \\
\text { ción nacional }\end{array}$} & I & - & 16,2 & 2,7 & - & - & - & - \\
\hline & $\mathrm{P}$ & - & 8,1 & - & - & - & 2,7 & - \\
\hline & $\mathrm{O}$ & - & - & 2,7 & - & - & 13,5 & - \\
\hline & I-P & - & 10,8 & - & - & - & - & - \\
\hline & $\mathrm{I}-\mathrm{O}$ & - & 8,1 & - & - & - & 10,8 & - \\
\hline & $\mathrm{P}-\mathrm{O}$ & - & 2,7 & - & - & - & 13,5 & - \\
\hline & $\mathrm{I}-\mathrm{P}-\mathrm{O}$ & - & 2,7 & - & - & - & 5,4 & - \\
\hline \multirow{7}{*}{$\begin{array}{l}\text { 27. Ingresos por proyectos investiga- } \\
\text { ción europea }\end{array}$} & I & - & 13,9 & 2,8 & - & - & 2,8 & - \\
\hline & $\mathrm{P}$ & - & 8,3 & - & - & - & - & - \\
\hline & $\mathrm{O}$ & - & - & - & - & - & 13,9 & - \\
\hline & $\mathrm{I}-\mathrm{P}$ & - & 8,3 & - & - & - & - & - \\
\hline & $\mathrm{I}-\mathrm{O}$ & - & 11,1 & - & - & - & 2,8 & - \\
\hline & $\mathrm{P}-\mathrm{O}$ & - & 2,8 & - & - & 2,8 & 22,2 & - \\
\hline & I-P-O & - & 8,3 & - & - & - & - & - \\
\hline \multirow{7}{*}{$\begin{array}{l}\text { 32. } N^{\mathrm{o}} \text { contratos de investigación } \\
\text { (universidades con entidades) }\end{array}$} & I & - & 2,9 & 2,9 & - & - & - & - \\
\hline & $\mathrm{P}$ & - & 5,7 & 2,9 & - & - & - & - \\
\hline & $\mathrm{O}$ & - & - & 8,6 & - & - & 31,4 & - \\
\hline & I-P & - & - & - & - & - & 2,9 & - \\
\hline & $\mathrm{I}-\mathrm{O}$ & - & 2,9 & - & - & - & 11,4 & - \\
\hline & $\mathrm{P}-\mathrm{O}$ & - & 2,9 & - & - & - & 14,3 & - \\
\hline & $\mathrm{I}-\mathrm{P}-\mathrm{O}$ & - & - & - & - & - & 11,4 & - \\
\hline \multirow{7}{*}{ 33. $\mathrm{N}^{\circ}$ de patentes solicitadas } & I & - & - & - & - & - & - & - \\
\hline & $\mathrm{P}$ & - & 5,4 & - & - & - & 5,4 & - \\
\hline & $\mathrm{O}$ & - & - & 35,1 & - & - & 29,7 & - \\
\hline & $\mathrm{I}-\mathrm{P}$ & - & - & - & - & - & - & - \\
\hline & $\mathrm{I}-\mathrm{O}$ & - & - & - & - & - & - & - \\
\hline & $\mathrm{P}-\mathrm{O}$ & - & - & 13,5 & - & - & 10,8 & - \\
\hline & $\mathrm{I}-\mathrm{P}-\mathrm{O}$ & - & - & - & - & - & - & - \\
\hline
\end{tabular}


TABLA V (continuación)

\begin{tabular}{c|c|c|c|c|c|c|c|c}
\hline \multirow{2}{*}{ Indicador } & \multirow{2}{*}{ Tipo+ } & \multicolumn{7}{c}{ Misión* } \\
\cline { 3 - 10 } & & D & I & TC & D-I & D-TC & I-TC & D-I-TC \\
\hline \multirow{3}{*}{$34 . N^{\circ}$ de licencias concedidas } & $\mathrm{I}$ & - & - & - & - & - & - & - \\
\cline { 2 - 10 } & $\mathrm{P}$ & - & - & - & - & - & - & - \\
\cline { 2 - 10 } & $\mathrm{O}$ & - & 2,7 & 54,1 & - & - & 27,0 & - \\
\cline { 2 - 9 } & $\mathrm{I}-\mathrm{P}$ & - & - & - & - & - & - & - \\
\cline { 2 - 9 } & $\mathrm{I}-\mathrm{O}$ & - & - & 2,7 & - & - & - & - \\
\cline { 2 - 9 } & P-O & - & - & 5,4 & - & - & 8,1 & - \\
\hline & I-P-O & - & - & - & - & - & - & - \\
\hline
\end{tabular}

Fuente: elaboración propia.

* (D) Docencia; (I) Investigación; (TC) Transferencia de Conocimiento; (D-I) Docencia e Investigación; (DTC) Docencia y Transferencia de Conocimiento; (I-TC) Investigación y Transferencia de Conocimiento; (D-I-TC) Docencia, Investigación y Transferencia de Conocimiento.

+ (I) Input; (P) Proceso; (O) Output; (I-P) Input y Proceso; (I-O) Input y Output; (P-O) Proceso y Output; (I-P-O) Input, Proceso y Output.

Finalmente, las observaciones realizadas por los panelistas nos informan que los expertos están totalmente de acuerdo en dos aspectos que parecen estar relacionados: los indicadores como método de evaluación deberían combinarse con métodos cualitativos y debería extenderse dicho método en los próximos años. Además, el grupo está de acuerdo en que es posible desarrollar un sistema de indicadores que refleje convenientemente las misiones de la universidad, sin suponer costes elevados por lo que este tipo de evaluación debería promoverse. Asimismo, el panel participante en la investigación se muestra en desacuerdo con respecto a que cada universidad desarrolle su propio sistema de indicadores. Por tanto, estas respuestas ponen de manifiesto que los gestores universitarios son conscientes de las ventajas de los indicadores como método de evaluación de las universidades públicas, y consideran que debería existir un acuerdo con respecto a un modelo único del mismo. Si bien también reconocen sus limitaciones y de ahí que se deba complementar con métodos cualitativos.

\section{Conclusiones}

El presente estudio contribuye a esclarecer el debate abierto en torno al establecimiento de criterios de clasificación para los indicadores utilizados en la evaluación de las IES, sobre el cual no existe consenso hasta la fecha. El centro de interés de este trabajo ha sido lograr cierto consenso entre un grupo de expertos que ocupan cargos académicos y de gestión y con amplios conocimientos en la evaluación de las instituciones universitarias, particularmente de las 
universidades españolas. Para ello, hemos aplicado el método Delphi con el propósito de identificar los indicadores que permitan evaluar la actividad universitaria.

Nuestros resultados muestran, en primer lugar, el consenso alcanzado entre los expertos con relación a la utilidad y validez del uso de indicadores en la evaluación de las universidades. Los resultados del Delphi ponen de manifiesto que los gestores universitarios consideran que esta herramienta es adecuada para realizar comparaciones entre universidades, para evaluar las IES y que por ello, debería extenderse en los próximos años. Sin embargo, se debe tener en cuenta sus limitaciones, por lo que también consideran que debería combinarse con otros métodos cualitativos de evaluación para realizar una evaluación óptima que ofrezca información fiable que facilite la toma de decisión sobre políticas de educación superior. De hecho, los resultados muestran que continúa habiendo discrepancias en cuanto a la identificación de dichos indicadores, tanto en la misión que representan como en su tipología y su grado de importancia.

Con respecto a la identificación de los indicadores para evaluar las misiones universitarias, los resultados muestran, por un lado, que algunos indicadores serían exclusivos para evaluar una misión y, por otro lado, que otros indicadores se utilizarían para evaluar más de una misión. Los indicadores claramente identificados con una misión son: 1) los orientados a la docencia, como el número de estudiantes en $1 .^{\text {er }}$ y $2 .{ }^{\circ}$ ciclo, el número de graduados y los resultados de la encuesta de evaluación de la labor docente del PDI; 2) los orientados a la investigación, como el número de publicaciones en revistas indexadas, el número de citas a dichas publicaciónes y el número de sexenios concedidos, y 3) los orientados a la transferencia de conocimiento, como la existencia de incubadora, el número de empresas en la incubadora y la participación en empresas que desarrollan actividades productivas basadas en la I+D universitaria.

Asimismo, conviene destacar el sesgo existente en los indicadores de transferencia de conocimiento ya que, principalmente, se refieren a las actividades que las OTRI vienen desarrollando, olvidando las actividades de transferencia más relacionadas con la divulgación científica y no tanto con la comercialización de los resultados de la investigación. Esta desviación debería tenerse en cuenta dado que las universidades transfieren su conocimiento a la sociedad a través de distintos mecanismos, algunos de los cuales son de difícil valoración. Por tanto, sería aconsejable alcanzar el consenso con relación a un listado de indicadores relacionados con la transferencia de conocimiento que incluya tanto las actividades que las OTRI gestionan como aquellas actividades que muchos académicos realizan para transferir su conocimiento.

Ocurre algo parecido si nos fijamos en la categorización que hacen los expertos participantes con relación a la tipología del indicador, es decir, si se trata de inputs, procesos u outputs. En este caso el consenso es menor entre los expertos. Solo 15 indicadores fueron considerados indicadores de output, mientras 2 indicadores fueron catalogados indicadores de procesos. Por tanto, especialmente destaca la dificultad de asignar la etiqueta de input y proceso a los indi- 
cadores presentados. Los indicadores en los que se ha observado mayor consenso hacen referencia a los resultados de la actividad universitaria relacionada con el número de graduados y postgraduados, los ingresos medios de los graduados en su primer trabajo y el número de citas que ha recibido una publicación.

En cuanto a la identificación de la importancia del indicador en el proceso de evaluación se observó un consenso aún menor, solo en 12 de ellos se alcanzó el acuerdo entre los expertos. Los resultados sugieren que aquellos indicadores considerados como los más importantes deberían ser incluidos en los procesos de evaluación de las universidades, como por ejemplo: el número de publicaciones en revistas indexadas, las citas recibidas por estas publicaciones, los ingresos por proyectos de investigación local y regional, nacional y europea, el número de licencias concedidas, el número de contratos de investigación, las patentes solicitadas, el número de graduados, el número de postgraduados que participan en actividades de I+D y el cociente de PDI por estudiante.

Asimismo, nuestro estudio confirma el extenso debate acerca de las consecuencias de incluir entre las misiones institucionales de la universidad, además de la docencia y la investigación, la transferencia de conocimiento. En este sentido, la transferencia de conocimiento podría ser vista como un problema de complementariedad versus sustitución en los outputs de la actividad universitaria, por ejemplo, el número de publicaciones en revistas indexadas, el número de contratos de la universidad con la industria y el número de patentes solicitadas. Por otro lado, podría considerarse un problema de complementariedad versus sustitución en la relación input-output dada la falta de consenso en torno a la consideración de los ingresos obtenidos por proyectos de investigación local y regional, nacional y europea. O finalmente, un problema de complementariedad versus sustitución en la relación proceso-output como muestra la respuesta de los expertos, por ejemplo, respecto al número de graduados que participan en actividades de I+D.

Nuestros resultados muestran, por tanto, que las actividades universitarias deben ser vistas como un vector de resultados producidos de forma conjunta mediante un mismo vector de recursos, como por ejemplo el número de PDI y el número de PAS. Desde esta perspectiva, se podría encontrar una correlación positiva, negativa o incluso mixta entre las actividades universitarias. Por tanto, el estudio de los sistemas de evaluación debe centrarse en la importancia de evaluar el desempeño de las universidades a nivel institucional con el propósito de analizar la posible relación de complementariedad o sustitución entre las misiones de docencia, investigación y transferencia de conocimiento.

A tenor de los resultados alcanzados, parece claro que los gestores universitarios están de acuerdo en la validez de los indicadores como un instrumento útil para la evaluación de las universidades. La puesta en marcha del método Delphi, mediante el cual hemos solicitado información a expertos que tienen experiencia en tema de evaluación institucional, se puede concluir que este trabajo contribuye, de forma teórica y práctica, en la priorización de indicadores para evaluar a las universidades. Sin embargo, persiste la necesidad de que las 
administraciones públicas implicadas y la comunidad universitaria en su conjunto alcancen un mayor consenso, siendo necesaria una mayor reflexión en torno a la definición de un modelo teórico de mínimos aceptado, que permita a las instituciones universitarias asignar pesos a los distintos indicadores en función de su planificación estratégica. Para tal fin, habría que mejorar la definición de cada uno de los indicadores, al igual que su medición. Sería recomendable avanzar en la estandarización de un manual de indicadores para evaluar las instituciones de educación superior, no solo a nivel nacional sino teniendo en cuenta el contexto europeo (European Commission, 2009).

Asimismo, sería necesario contar con una infraestructura estadística a nivel micro de los centros universitarios, coherente y transparente, que proporcionara a estas instituciones y a los responsables políticos información pertinente para evaluar comparativamente y realizar un seguimiento de las tendencias que desarrolla el sector de la educación superior, el cual está acometiendo una transformación y expansión sustancial que requiere y exige ser supervisada y evaluada para facilitar la toma de decisiones y adquirir un mayor conocimiento de la rentabilidad económica y social de la inversión en educación superior. Si el acuerdo fuera alcanzado, podría construirse una estructura permanente encargada de la recogida sistemática y periódica de datos normalizados con una metodología bien desarrollada, que permitiría desarrollar evaluaciones de las universidades en base a un método comúnmente aceptado.

\section{Agradecimientos}

Este trabajo ha recibido apoyo del proyecto ref. ECO2008-02553/ECON, titulado "Las misiones de la universidad y su complementariedad. Nuevos métodos de evaluación en términos de eficiencia», financiado por el Plan Nacional de I+D del Ministerio de Ciencia e Innovación.

\section{Referencias}

Ahn, T.; Charnes, A., y Cooper, W. W. (1988). Using DEA to measure the efficiency of non-for-profit organizations: A critical evaluation-comment. Managerial and Decision Economics, vol. 9 (3), 251-253.

Ahn, T., y Seiford, L. W. (1993). Sensitivity of DEA to models and variable sets in a hypothesis test setting: The efficiency of university operations. En: Ijiri, I. (ed.) Creative and Innovative Approaches to the Science of Management. Quorum Book, Westport CT.

AQU (2007). El Sistema Universitari Públic Català 2000-2005: Una Perspectiva des de l'avaluació d'AQU Cataluña. Barcelona; Agencia para la Calidad del Sistema Universitario Catalán.

Beasley, J. E. (1995). Determining teaching and research efficiencies. Journal of the Operational Research Society, vol. 46 (4), 441-452. 
Blank, R. K. (1993). Developing a System of Education Indicators: Selecting, Implementing and Reporting Indicators. Educational, Evaluation and Policy Analysis, vol. 15 (1), 65-80.

Bonaccorsi, A., y Daraio, C. (2007). Universities as strategic knowledge creators. Some preliminary evidence. En: Bonaccorsi, A., y Daraio, C. (eds.) Universities and Strategic Knowledge Creation. Edward Elgar Publishing Ltd, UK.

Bonaccorsi, A.; Daraio, C.; Lepori, B., y Slipersaeter, S. (2007). Indicators on individual higher education institutions: addressing data problems and comparability issues. Research Evaluation, vol. 16 (2), 66-78.

Buela-Casal, G.; Gutiérrez-Martínez, O.; Bermúdez-Sánchez, M. P., y Vadillo-Muñoz, Ó. (2007). Comparative study of international academic rankings of universities. Scientometrics, vol. 71 (3), 349-365.

Buela-Casal, G.; Bermúdez, M. P.; Sierra, J. C.; Quevedo-Blasco, R., y Castro, A. (2009). Ranking de 2008 en productividad en investigación de las universidades públicas españolas. Psicothema, vol. 21 (2), 304-312.

Buesa, M.; Heijs, J., y Kahwash, O. (2009). La calidad de las universidades en España. Elaboración de un índice multidimensional. Madrid; CES.

Castro, A.; Buela-Casal, G. (2008). La movilidad de profesores y estudiantes en programas de postgrado: ranking de las universidades españolas. Revista de Investigación en Educación, vol. 5, 61-74.

CIHE (2007). Standards for Accreditation. Commission on Institutions of Higher Education. New England Association of Schools and Colleges. http://www.neasc.org/cihe/standards_for_accreditation_2005.pdf [24-04-07].

Cohn, E. R. S., y Santos, M. C. (1989). Institutions of higher education as multiproduct firms: Economies of scale and scope. Review of Economics and Statistics, vol. 71 (2), 284-290.

Dalkey, N., y Helmer, O. (1963). An experimental application of the Delphi method to the use of experts. Management Science, vol. 9 (3), 458-467.

D’Este, P.; Castro-Martínez, E., y Molas-Gallart, J. (2009). Documento de base para un "Manual de Indicadores de Vinculación de la universidad con el entorno socioeconómicon: un marco para la discusión. (1. ${ }^{a}$ ed.) Buenos Aires; OEI y AECID, p. 42.

ENQA (2009). Standards and guidelines for quality assurance in the European Higher Education Area. ENQA. Helsinki; European Association for Quality Assurance in Higher Education, p. 48.

Escrigas, C. (2006). Perspectivas de los líderes sobre la financiación de la educación superior. Encuesta Delphi. En: GUNI (ed.) La educación superior en el mundo 2006: La financiación de las universidades. Mundiprensa, Barcelona.

European Commission (2005). European Universities: Enhancing Europe's Research Base. Brussels; European Commission, p. 48.

European Commission (2009). EUMIDA Project. h ttp://www.eumida.org [29.09.2010].

Fundación CyD (2011). La contribución de las universidades españolas al desarrollo. Informe CyD 2010. Madrid; Fundación CyD, p. 384.

García-Aracil, A. (2007). Expansion and reorganization in the Spanish higher education system. En: Bonaccorsi, A.; Daraio, C. (editores) Universities and Strategic Knowledge Creation. Edward Elgar Publishing Ltd, UK. 
García-Aracil, A., y Palomares-Montero, D. (2010). Examining benchmark indicator systems for the evaluation of higher education instittutions. Higher Education, vol. 60 (2), 217-234.

Geist, M. R. (2010). Using the Delphi method to engage stakeholders: A comparison of two studies. Evaluation and Program Planning, vol. 33 (2), 147-154.

Gibbons, M. (1999). Science's new social contract with society. Nature, vol. 402 (6761), $11-18$

GUNI (editores) (2007). La educación superior en el mundo 2007: Acreditación para la garantía de la calidad ¿qué está en juego? Barcelona; Mundiprensa, p. 464.

Gustafso, D. H.; Shukla, R. K., Delbecq, A., y Walster, G.W. (1973). Comparative study of differences in subjective likelihood estimates made by individuals, interacting groups, Delphi groups and nominal groups. Organizational Behavior and Human Performance, vol. 9 (2), 280-291.

Hanke, M.; Leopoldseder, T. (1998). Comparing the efficiency of Austrian universities. A data envelopment analysis application. Tertiary Education and Management, vol. 4 (3), 191-197.

Hernández Armenteros, J. (2003). Universidades e Indicadores de Gestión: El documento de la Conferencia de Rectores de las Universidades Españolas. Año 2000. Jornadas "Indicadores sobre la calidad universitaria". Madrid, España: Universidad Carlos III.

Johnes, J., y Johnes, G. (1995). Research funding and performance in UK university departments of economics: A frontier analysis. Economics of Education Review, vol. 14 (3), 301-314.

Kells, H. R. (1991). The inadequacy of performance indicator for higher education: the need for a more comprehensive and development construct. Higher Education Management, vol. 2 (3), 258-270.

Kim, Y. H., y Kim, H. H. (2008). Development and validation of evaluation indicators for a consortium of institutional repositories: A case study of dCollection. Journal of the American Society for Information Science and Technology, vol. 59 (8), 1282-1294.

Landeta, J. (2006). Current validity of the Delphi method in social sciences. Technological Forecasting and Social Change, vol. 73 (5), 467-482.

Landeta, J. (1999). El método Delphi. Una técnica de previsión para la incertidumbre. Barcelona; Ariel, p. 224.

Laredo, P. (2007). Revisiting the third mission of universities: toward a renewed categorization of university activities? Higher Education Policy, vol. 20 (4), 441-456.

Lepori, B.; Barré, R., y Filliatreau, G. (2008). New perspectives and challenges for the design and production of S\&T indicators. Research Evaluation, vol. 17 (1), 33-44.

Lobera, J. (2008). Encuesta Delphi: la educación superior para el desarrollo humano y social. En: GUNI (ed.) La educación superior en el mundo: nuevos retos y roles emergentes para el desarrollo bumano y social. Mundiprensa, Madrid.

Martin, B.; Etzkowitz, H. (2000). The origin and evolution of the university species. VEST, vol. 13 (3-4), 9-34.

Molas-Gallart, J. (2002). Measuring Third Stream Activities. Brighton; University of Sussex, 97.

Molas-Gallart, J., y Castro-Martínez, E. (2007). Ambiguity and conflict in the development of «Third Mission» indicators. Research Evaluation, vol. 16 (4), 321-330. 
Oakes, J. (1989). What Educational Indicators? The case for assessing the school context. Educational, Evaluation and Policy Analysis, vol. 11 (2), 181-199.

Okoli, C., y Pawlowski, S. D. (2004). The Delphi method as a research tool: an example, design considerations and applications. Information \& Management, vol. 42 (1), 15-29.

Palomares-Montero, D.; García-Aracil, A., y Castro-Martínez, E. (2008). Evaluación de las instituciones de educación superior: revisión bibliográfica de sistema de indicadores. Revista Española de Documentación Científica, vol. 31 (2), 205-229.

Rowe, G., y Wright, G. (1999). The Delphi technique as a forecasting tool: issues and analysis. International Journal of Forecasting, vol. 15 (2), 353-375.

Santana Armas, F. (2007). Las políticas de evaluación de las universidades vistas por un grupo de expertos. En: Giró Miranda, J. (coord.) La escuela en el siglo XXI: la educación en un tiempo de cambio social acelerado. Universidad de La Rioja, Logroño.

Sinuany-Stern, Z.; Mehrez, A., y Barboy, A. (1994). Academic departments efficiency via DEA. Computers Operations Research, vol. 21 (5), 543-556.

Sizer, J. (1982). Institutional performance assessment under conditions of changing needs. International Journal of Institutional Management in Higher Education, vol. 6 (1), $17-28$.

Von der Gracht, H. A. (2008). The Delphi Technique for Future Research. En: von der Gracht, H. A. (ed.) The future of logistics. Scenarios for 2025. GWV Fachverlage Gmbh, Wiesbaden.

Worthington, A. C. (2001). An empirical survey of frontier efficiency measurement techniques in education. Education Economics, vol. 9 (3), 245-268. 


\title{
APÉNDICE 1
}

Encuesta Delphi: «El uso de indicadores en la evaluación de universidades» (VERSIÓN FINAL)

\author{
I. VARIABLES SOCIO-DEMOGRÁFICAS \\ GÉNERO: \\ AÑO DE NACIMIENTO: \\ CATEGORIA PROFESIONAL ACTUAL \\ TIPO DE INSTITUCIÓN: \\ ÁREA DE CONOCIMIENTO ANEP en la que desarrolla su principal actividad investigadora:
}

\section{OPINIÓN GENERAL DEL EXPERTO RESPECTO AL USO DE INDICADORES EN LA EVALUACIÓN DE UNIVERSIDADES}

Por favor, exprese su opiniỏn en una escala de (1) "Totalmente en desacuerdo" a (5) "Totalmente de acuerdo" sobre las siguientes afirmaciones relacionadas con el uso de indicadores en la evaluación de las universidades.

\begin{tabular}{|c|c|}
\hline & $\begin{array}{l}\text { 1- Totalmente en desacuerdo } \\
5 \text { - Totalmente de acuerdo } \\
\text { (respuesta simple) }\end{array}$ \\
\hline Es posible desamolar un sistema de indicadores que refleje convenientemente las misiones de la universidad & $\begin{array}{lllll}1 & 2 & 3 & 4 & 5 \\
\square & \square & \square & 口 & \square\end{array}$ \\
\hline Es posible llevar a cabo comparaciones entre las universidades utilizando sistemas de indicadores & $\begin{array}{lllll}1 & 2 & 3 & 4 & 5 \\
\square & \square & \square & \square & \square \\
\end{array}$ \\
\hline El uso de indicadores como método de evaluación de universidades deberia combinarse con métodos cualitativos & $\begin{array}{lllll}1 & 2 & 3 & 4 & 5 \\
\text { ㅁ } & \text { ㅁ } & \text { 口 } & \text { 口 } & \text { 口 } \\
\end{array}$ \\
\hline $\begin{array}{l}\text { El uso de indicadores en la evaluación de universidades no supone costes elevados por lo que este fipo de evaluación } \\
\text { deberia promoverse }\end{array}$ & $\begin{array}{lllll}1 & 2 & 3 & 4 & 5 \\
\square & \square & \square & \square & \stackrel{\square}{\square} \\
\end{array}$ \\
\hline Cada universidad debe desarnollar su propio sistema de indicadores & $\begin{array}{lllll}1 & 2 & 3 & 4 & 5 \\
\square & \square & \square & \square & \square \\
\end{array}$ \\
\hline El uso de indicadores como método de evaluación de uriversidades se extenderá en los proximos 5 años & $\begin{array}{lllll}1 & 2 & 3 & 4 & 5 \\
\text { 口 } & \text { 口 } & \text { 口 } & \text { 口 } & \text { 口 } \\
\end{array}$ \\
\hline
\end{tabular}

III. IDENTIFICACIÓN DE INDICADORES UTILIZADOS EN LA EVALUACIÓN DE UNIVERSIDADES.

A continuación se presenta una serie de indicadores que están recopilando diferentes organismos oficiales para evaluar las universidades. Por favor, marcando con una $\mathbf{\otimes}$ su respuesta, exprese su opinión sobre los siguientes aspectos:

- Los indicadores informan sobre la:

(D) Docencia (transmisión de conocimientos y destrezas a los estudiantes a través de la formación y de los procesos de capacitación)

(I) Investigación (generación de nuevo conocimiento a través del análisis y la experimentación)

(TC) Transferencia de Conocimiento (aplicación y explotación de conocimiento fuera del contexto académico)

- Los indicadores reflejan los:

(l) Inputs (Recursos de las Universidades)

(P) Proceso (Actividad de las Universidades)

(O) Outputs (Resultados de las Universidades)

- ¿Cuál es la importancia que usted da al indicador en el proceso de evaluación?:

Exprese su opinión en una escala de (1) "Nada importantex (no debe ser considerado en el proceso de evaluación) a (5) "Muy importante" (esencial en el proceso de evaluación)

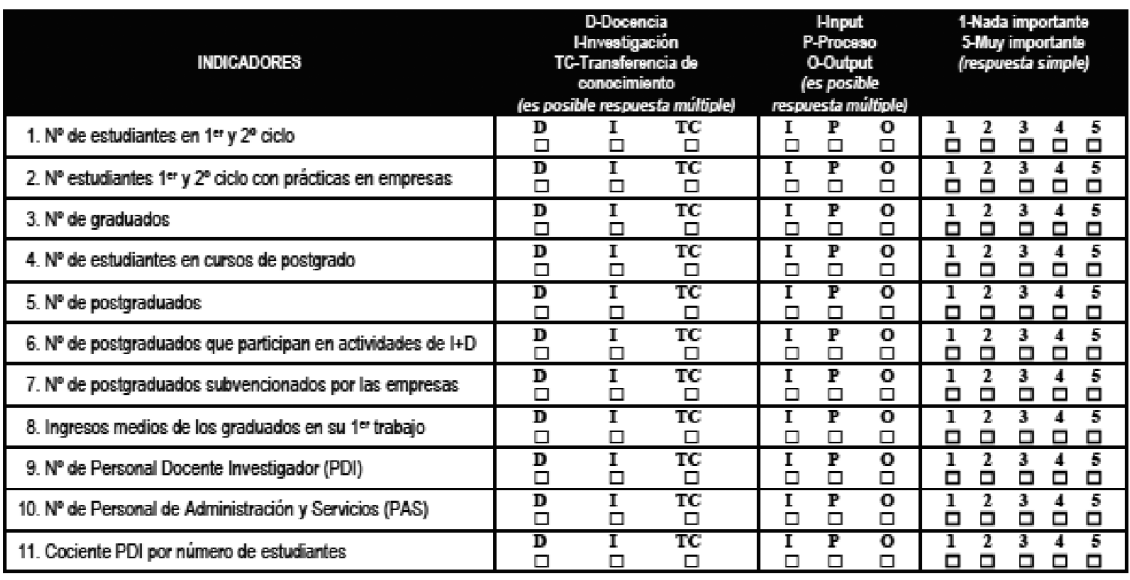




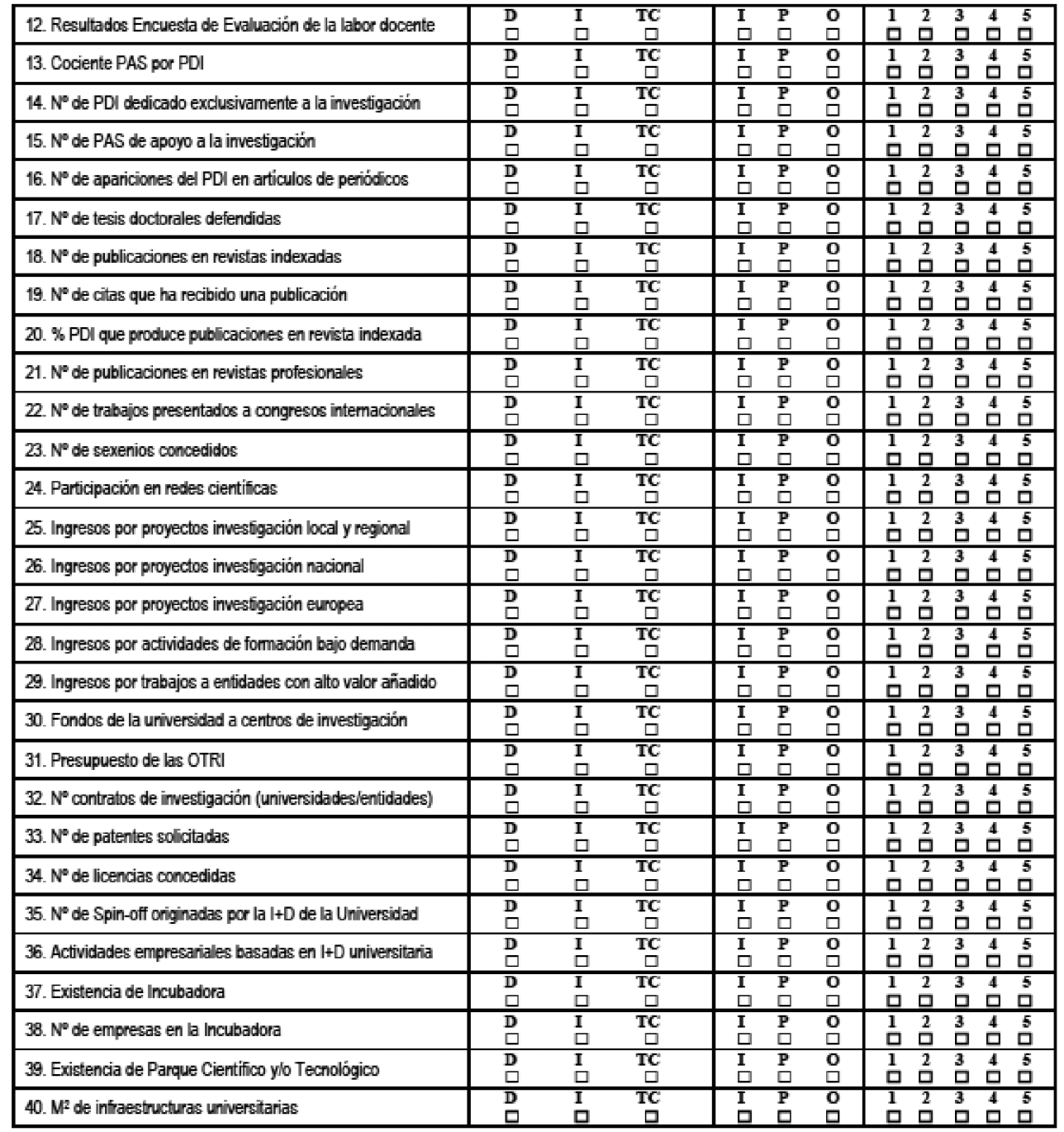

OBSERVACIONES:

Si tiene algün comentario o sugerencia en relación a esta sección, por favor indiquelo aquí

Si tiene algún comentario o sugerencia sobre la encuesta Delphi, por favor indiquelo aquí 